\title{
Conceptual design of a wide-field near UV transient survey in a $6 U$ CubeSat
}

\section{Yoichi Yatsu, Toshiki Ozawa, Kenichi Sasaki, Hideo Mamiya, Nobuyuki Kawai, et al.}

Yoichi Yatsu, Toshiki Ozawa, Kenichi Sasaki, Hideo Mamiya, Nobuyuki Kawai, Yuhei Kikuya, Masanori Matsushita, Saburo Matunaga, Shouleh Nikzad, Pavaman Bilgi, Shrinivas R. Kulkarni, Nozomu Tominaga, Masaomi Tanaka, Tomoki Morokuma, Norihide Takeyama, Akito Enokuchi, "Conceptual design of a wide-field near UV transient survey in a $6 \mathrm{U}$ CubeSat," Proc. SPIE 10699, Space Telescopes and Instrumentation 2018: Ultraviolet to Gamma Ray, 106990D (6 July 2018); doi: 10.1117/12.2313026 United States 


\title{
Conceptual design of a wide-field near UV transient survey in a $6 \mathrm{U}$ CubeSat
}

Yoichi Yatsu $^{\mathrm{a}}$, Toshiki Ozawa ${ }^{\mathrm{a}}$, Kenichi Sasaki ${ }^{\mathrm{b}}$, Hideo Mamiya ${ }^{\mathrm{a}}$, Nobuyuki Kawai ${ }^{\mathrm{a}}$, Yuhei Kikuya $^{b}$, Masanori Matsushita ${ }^{b}$, Saburo Matunaga ${ }^{b}$, Shouleh Nikzad ${ }^{d}$, Pavan Bilgi ${ }^{c}$, Shrinivas R. Kulkarni ${ }^{\mathrm{e}}$, Nozomu Tominaga ${ }^{\mathrm{f}}$, Masaomi Tanaka ${ }^{\mathrm{g}}$, Tomoki Morokuma ${ }^{\mathrm{h}}$, Norihide Takeyamai, and Akito Enokuchi ${ }^{i}$

aDept of Physics, Tokyo Institute of Technology, 2-12-1 Ohkayama, Meguro, Tokyo 152-8551, Japan

${ }^{b}$ Dept. of Mechanical Engineering, Tokyo Institute of Technology, Meguro, Tokyo, Japan

d Jet Propulsion Laboratory, California Institute of Technology, 4800, Oak Grove Drive, Pasadena, CA 91109, USA

${ }^{\mathrm{c}}$ Dept. of Aerospace, California Institute of Technology, 1200 East California Boulevard, Pasadena, CA 91125, USA

eDivision of Physics, Math and Astronomy, California Institute of Technology, 1200 East California Boulevard, Pasadena, CA 91125, USA

${ }^{\mathrm{f}}$ Dept. of Physics, Konan University, 8-9-1, Okamoto, Higashinada, Kobe, Hyogo 658-0072, JAPAN

${ }^{\text {g} D e p t . ~ o f ~ A s t r o p h y s i c s, ~ T o h o k u ~ U n i v e r s i t y, ~ 6-3, ~ A r a m a k i ~ A z a-A o b a, ~ A o b a-k u, ~ S e n d a i ~}$ 980-8578, JAPAN

h Institute of Astronomy, University of Tokyo, 2-21-1, Osawa, Mitaka, Tokyo 181-0015, JAPAN ${ }^{\mathrm{i}}$ GENESIA Corporation, Mitaka, 3-38-4-601, Shimorenjaku, Mitaka, Tokyo 181-0013, JAPAN

\begin{abstract}
A conceptual design of a wide-field near UV transient survey in a $6 \mathrm{U}$ CubeSat is presented. Ultraviolet is one of the frontier in the transient astronomy. To open up the discovery space, we are developing a $6 \mathrm{U}$ CubeSat for transient exploration. The possible targets will be supernova shock-breakouts, tidal disruption events, and the blue emission from NS-NS mergers in very early phase. If we only focused on nearby/bright sources, the required detection limit is around $20 \mathrm{mag}(\mathrm{AB})$. To avoid the background and optical light, we chose a waveband of 230-280 nm. As an imaging detector, we employ a delta-doped back-illuminated CMOS. In addition to delta doping, the multi-layer coating directly deposited on the detector enables both a high in-band UV QE and the ultra-low optical rejection ratio. Taking into account these specifications, even an $8 \mathrm{~cm}$ telescope can achieve the detection limit of 20 mag $_{\mathrm{AB}}$ for a FoV larger than $60 \mathrm{deg}^{2}$. We also designed the satellite bus system for this mission by utilizing commercially available cubesat components satisfying the mission requirements.
\end{abstract}

Keywords: Ultraviolet, time-domain, cubesat, gravitational wave, supernovae shockbreakouts, Tidal disruption events

\section{INTRODUCTION}

Ultraviolet (UV) is the one of the last frontiers in the electromagnetic (EM) astronomy. Since 2010, the time domain and multi-messenger astronomy has been perceived as a promising discovery space to find unknown phenomena or objects in universe. The most symbolic target has been the gravitational wave $(\mathrm{GW})$ sources in this decade. The progress of interferometers, such as LIGO, Virgo and KAGRA, has motivated astronomers strongly to develop and automatize observational systems including data analysis pipelines to detect the EM

Y.Y.: E-mail: yatsu@hp.phys.titech.ac.jp, Telephone: +81 357342388

Space Telescopes and Instrumentation 2018: Ultraviolet to Gamma Ray, edited by Jan-Willem A. den Herder, Shouleh Nikzad, Kazuhiro Nakazawa, Proc. of SPIE Vol. 10699, 106990D · (c) 2018 SPIE CCC code: $0277-786 X / 18 / \$ 18 \cdot$ doi: $10.1117 / 12.2313026$ 
counterparts of GW sources promptly. As a result, those efforts led us to the successful detection of the EM counterpart of GW170817 in 2017. ${ }^{1-3}$ There were several theoretical models predicting red/IR emission due to the existence of neutron-rich material synthesized in the merging process of the neutron star (NS)-NS binary. However the discovered EM counterpart showed rather blue emission in the early phase before showing redder emission due to the kilonova (macronova). At this moment, no one knows the mechanism of the blue emission in the early phase however, this will provide crucial information of the dynamics of merging process and motion of ejecta, that will be related to the origin of short gamma-ray bursts (sGRBs). Moreover the bluer band is convenient to find the EM counterpart in the early phase within $10 \mathrm{hr}$ from the GW detection. This means that an UV wide-field exploration mission will have significant advantages in GW counterpart survey and also in the other sciences.

In spite of the scientific potential as shown above, the UV sky has not been observed with wide-field imagers due to technical difficulties. In fact there have been several space telescopes for near UV band (NUV), OM/XMM-Newton, WFC3/HST, GALEX, UVOT/Swift, andUVIT/Astrosat. Among the above modern UV space telescopes, GALEX has the largest FoV with a diameter of $\phi 1.2^{\circ}$, which led the detection of an UV flash interpreted to be the direct evidence of a shock breakout going in advance of core-collapse supernova. The first burrier for the UV survey is atmosphere, therefor we cannot observe NUV light bluer than $~ 300 \mathrm{~nm}$ on the ground. Second obstacle is background. Most of stars are emitting optical light which must be rejected when we observe NUV band. Therefore the detector must have a high UV-transmittance and solar-blind filter. The final burrier is the dead layer of imaging detector. Due to the short absorption length of UV light, the incident photons are photo-absorbed just behind the surface from which most of photo-electrons cannot be collected by the electrodes.

To open-up the unprecedented discovery space, we propose a path-finder mission utilizing a $6 \mathrm{U}$ cubesat platform. We have developed micro-satellites since 2000 for technology demonstrations and space sciences. ${ }^{4}$ Nowadays, we can purchase cheap and high-performance satellite components via the internet. Utilizing those things, we can develop a satellite system much earlier, easier and cheaper. To pack the instrument in the very small satellite, we must shrink the size of telescope with no change in sensitivity. One of the solutions to overcome those technical problems are "Delta-doping" and an anti-reflection (AR) coatings directly deposited on detector surface developed by the Microdevices Laboratory/JPL, that enables quite high quantum efficiency even in UVband. ${ }^{5}$ In this paper, we briefly describe the scientific motivation and then breakdown the mission requirements $(\S 2)$. Based on the given constraints, we design the the mission instrument and estimate the performance (§3). To fullfil the mission requirements, we then design the satellite bus system by using commercially available satellite components $(\S 4)$.

\section{MISSION ANALYSIS}

\subsection{Targets}

Here we briefly summarize the scientific motivation and the specification of the potential targets of this mission.

\section{Gravitational Wave sources (NS-NS mergers)}

As we mentioned in $\S 1$, we are greatly interested in the merging process of NS-NS binaries. UV emission may provide the information on the dynamics of high electron fraction, $Y_{\mathrm{e}}{ }^{*}$, ejecta or free neutrons survived under the rapid electron capturing process. ${ }^{6,7}$ Furthermore, the distributions of high- $Y_{\mathrm{e}}$ material can explain the relation between the NS-NS mergers and sGRBs of which origins are still unclear. At this moment, we have only one sample of NS-NS merger appeared at $\sim 47 \mathrm{Mpc}$ away from the earth. ${ }^{1}$ Taking into account the measured SED evolution of GW170817, ${ }^{8}$ the expected brightness of NS-NS merger at a distance of $200 \mathrm{Mpc}$ which is the observable range of $a L I G O$, would be still brighter than $\sim 20$ mag in NUV-band at 0.5 day after the merger. The predicted UV emission from the free neutron decay can be much brighter than that of typical kilonova but the duration will be shorter than an hour. ${ }^{7}$ To detect this intriguing emission, we must start observation within an hour from a GW alert.

\footnotetext{
${ }^{*}$ The electron fraction is defined by $Y_{\mathrm{e}}=n_{\mathrm{p}} /\left(n_{\mathrm{n}}+n_{\mathrm{p}}\right)$, where $n_{\mathrm{p}}$ and $n_{\mathrm{n}}$ are the densities of protons and neutrons, respectively.
} 


\section{Supernovae shock breakouts}

The final stage of the stellar evolution is another important issue to be solved by UV observations. GALEX discovered the first glimpse of supernova shock breakout(SNSB). ${ }^{9}$ The brightness of the core-collapse SNSBs can be crucial information constraining the physical size of the progenitors' photospheres. Tominaga et al. ${ }^{10}$ calculated the expected event rate of core-collapse SNe. The expected event rate for a field of view of $100 \mathrm{deg}^{2}$ at the detection limit of $20 \mathrm{mag}$ in NUV-band is $3 \sim 4$ events $\mathrm{yr}^{-2}$. Moreover the follow-up observations with ground-based telescopes provide the information on the historical activities in the terminal phase of massive stars. That can be also key information to solve the big question in astrophysics, namely the high-energy emission from long GRBs that seems to be strongly related to the distribution of stellar winds.

\section{Tidal Disruption Events}

When a star is orbiting near around a super massive black hole (SMBH) lying at the center of galaxy, the star will be disrupted by the strong tidal force. The teared stellar material construct an accretion disk around the $\mathrm{BH}$ and a jet which can be observed in the energy band from UV to soft X-rays. These activities may be related to the growth process of SMBHs that is one of the most mysterious questions to be solved in astronomy and cosmology. Detection of UV light and following observations can constrain the type of galaxies and stars which produce tidal disruption events. The radiation from those accretion disk continuing from several days to a year that is much longer than the above targets. The expected event rate for $100 \mathrm{deg}^{2}$ survey at a depth of $20 \mathrm{mag}$ in NUV band is $2 \sim 3 \mathrm{yr}^{-1} .11$

As might be expected, there are a lot of attractive potential UV transients, Type-Ia SNe, GRBs including their optical flashes, stellar flares etc. ${ }^{11,12}$ Table 1 summarize the event rate assuming a detection limit of 20 mag in NUV-band and a survey area of $100 \mathrm{deg}^{2}$.

Table 1. Summary of Targets.

\begin{tabular}{lccc}
\hline \hline Target & Position uncertainty & Duration & Event rate \\
\hline GWs (NS-NS) & $\sim 100 \mathrm{deg}^{2}$ & $0.5 \sim 10 \mathrm{hr} ?{ }^{7,8}$ & unknown $\left(>20 \mathrm{mag} @ 200 \mathrm{Mpc}^{8}\right)$ \\
SN SBs & Unpredictable & $\sim 0.5 \mathrm{hr} ?$ & $\sim 3 \mathrm{yr}^{-1} 100 \mathrm{str}^{-1} 10$ \\
TDEs & Unpredictable & a few days $\sim$ month & $\sim 2 \mathrm{yr}^{-1} 100 \mathrm{str}^{-1} 11$ \\
\hline
\end{tabular}

Note - Event rates are calculated with assumptions of a detection limit of 20 mag in NUV-band and a FoV of $100 \mathrm{deg}^{2}$.

\subsection{Mission Requirements}

Table 2 summarize the mission requirements to detect and measure the targets shown above. Among them, GW sources have the most critical requirements: a survey area of $\sim 100 \mathrm{deg}^{2}$ at a detection limit of 20 mag in NUV-band. Moreover those surveys must be completed within $\sim 1$ hour from the GW detection to clarify the UV emission mechanisms. While SNSBs make more strenuous requirements on the cadence to be higher than 2 $\mathrm{hr}^{-1}$, as their duration is quite short.

Another hard requirement for a small satellite bus is the constant connection to receive GW alerts from the ground. If we detect an UV transient, we must also downlink its coordinate, brightness and the other useful parameters as soon as possible to coordinate follow-up observations cooperating with ground-based observatories and the other satellites. The allowable communication delay is $\sim 30 \mathrm{~min}$ for both uplink and downlink to achieve the science goals.

The sky coverage is another issue. The angular distance of GW170817 from the sun was only $40^{\circ}$ that made the follow-up observations much harder. In fact, we gave up observation due to low elevation and twilight in Japan. If we observe this object a sky coverage of $\sim 11$ str is required. Although space telescopes which are outside the atmosphere can observe the sky near the sun, the telescope must have a huge baffle that will be 
several times larger than the $6 \mathrm{U}$ satellite itself. Moreover the satellite will have a critical (un-safe) attitude when it is pointing around the sun direction in the aspects of power and thermal managements. If we cannot have a baffle due to the limit of payload, the sky coverage will be smaller than $2 \pi$ str.

The observation efficiency depends on the orbit of the satellite. In UV-band, the earth-shine including OI line $(247.1 \mathrm{~nm})$ can be the major background in the dayside of the earth. To avoid this foreground UV photons, in-orbit observations should be scheduled only in the night side of the earth as in the case with GALEX. ${ }^{13}$ Although a twilight orbit can maximize the observation efficiency but the background is rather high and the half of the sky is always blocked by the earth. Therefore it is not worth the effort searching for rare chances to go to that orbit. There are a lot of flight chances to go to sun-synchronous low-earth orbits for remote sensing satellites. If we can get a ticket for the "regular flights", we can get UV data much earlier. In that case the observation efficiency will be lower than $50 \%$. In addition we must take into account the time for pointing. If we follow-up GW sources and the other interesting targets discovered, the satellite must have high performance, namely high slew-speed and highly stabilized, attitude control system. The dominant delay will be arise from communication disconnection, therefore the pointing delay should be negligibly short, within $\sim$ several minutes.

As a conclusion, we expect 3 SNSBs, 2 TDEs, as many as type-Ia SNe, and much more stellar flares in a year. That seems sufficient for a path-finder mission. Taking into account the man power of the satellite team, consists of 15 graduate students, the expected event rate seems reasonable. Considering the calibration phase, the mission life should be 3 years for surely achieving science goals. At least, our satellites that survived under critical phase have lived longer than 5 years.

Table 2. Summary of Mission Requirements.

\begin{tabular}{lr}
\hline \hline Energy band & NUV-band $(200 \sim 300 \mathrm{~nm})$ \\
Sensitivity & $\geq 20 \mathrm{mag}_{\mathrm{AB}}$ \\
Survey Area & $\leq 100 \mathrm{deg}^{2} \mathrm{scan}^{-1}$ \\
Cadence & $\leq 30 \mathrm{~min}$ \\
Sky Coverage & $\sim 2 \pi \mathrm{str}^{\dagger}$ \\
(Sun-avoidance Angle & $\left.\sim 90^{\circ}\right)$ \\
Observation efficiency & Higher is $\mathrm{Better}^{\dagger}$ \\
Pointing Delay (MNV speed) & $\leq 5 \mathrm{~min}$ \\
Communication Delay (Up \& Down) & $\leq 30 \mathrm{~min}$ \\
Launch & Earlier is Better \\
Mission Life & $\geq 3 \mathrm{yrs}$ \\
\hline
\end{tabular}

${ }^{\dagger}$ Those parameters are constrained by the main satellite.

\subsection{Mission Concept}

We aim to develop a pathfinder mission therefore our design concept is "Wide and Shallow" and should be launched earlier as soon as possible. That is the reason why we decided to utilize the cubesat platform. It is quite difficult to obtain useful information from far-distant and therefore dim sources with poor photon statistics. We cannot pack a large telescope into our satellite therefore the detected sources will be always bright which can be easily followed up with small telescopes on the ground.

Figure 1 describes the mission sequence invoked by a GW detection. If we receive an alert from the GW interferometers, we promptly uplink a command to start follow-up observation to the satellite via communication satellites such as IRIDIUM (Fig1-(1)). Then the satellite scans the error circle and takes images (Fig1-(2)). Because the data size of the short burst data of IRIDIUM is quite small, it is impossible to downlink the obtained images in real time. In order to announce the detection of the UV transient within the given allowable delay and to coordinate follow-up observations, the obtained images must be analyzed immediately in-orbit, and 
then the minimal compressed data of coordinate, magnitude and the other useful information are downlinked in real time (Fig1-(3)). Then we follow up the UV transient with observatories on ground and in space (Fig1-(4)). Subsequently, after the follow-up observations, the raw-data is downlinked to our ground stations in the visible pass of the satellite (Fig1-(5)). While in normal times, the satellite points around the anti-sun and high galacticlatitude region for exploring UV transients such as SNSBs, TDEs and the other sources. If the satellite detects transients, position information will be downlinked in the same way with GW events.

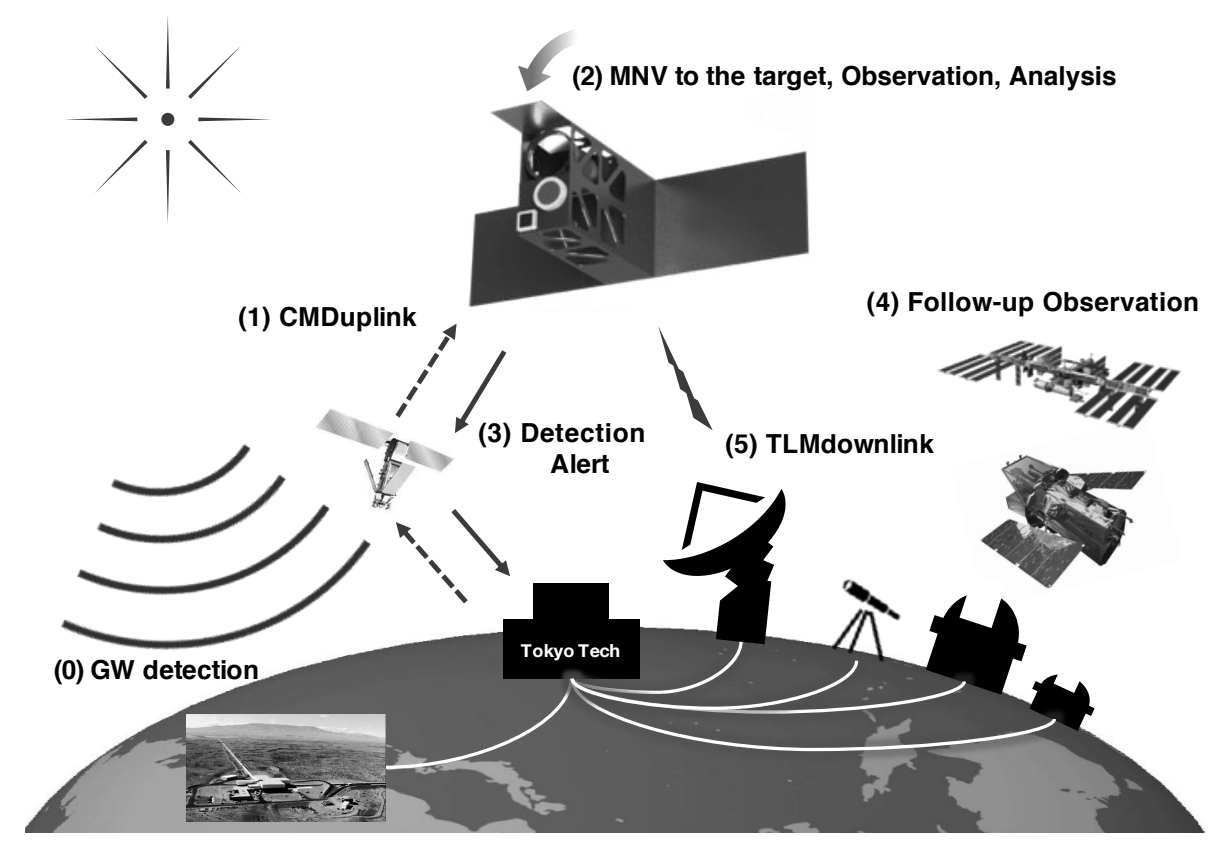

Figure 1. Schematic description of the observation sequence.

\section{MISSION INSTRUMENTS}

\subsection{Imager \& Filter}

The most critical issues to be solved is the development of imaging detector sensitive to UV-band. Moreover to obtain a wide field of view, the detector must be large in size. First we had surveyed commercially available detectors and found several high-sensitive BI-CCDs, however, those detectors require a long readout time and a low operation temperature of liquid nitrogen, that are inconvenient for cubesats. For those issues the micro device laboratory/JPL is developing a low-noise and large-area BI-CMOS imager for UV-band applying the Deltadoping technology. ${ }^{5}$ Another key technology for this mission is the direct deposition of AR coating on Si-wafer, that enables a high transmission ratio in UV-band with a quite low optical leakage below $10^{-3}$ simultaneously. ${ }^{14}$ Hereafter we adopt the dimension of detector, an array size of $4 \mathrm{k} \times 4 \mathrm{k}$ pixels with a pixel pitch of $12 \mu \mathrm{m}$ based on the preliminary design in prototyping.

\subsection{Optical System}

\subsubsection{Design constraints}

In order to realize the UV observation mission earlier, we choose to utilize the cubesat standard, for which various services not only flight components but also softwares, communication services and flight opportunities are available. At this moment the mainstream is $3 \mathrm{U}$ or $6 \mathrm{U}$ type container with the shortest side length of 116 $\mathrm{mm}$ which stringently constrain the size of telescope, namely the diameter of the primary mirror. 
At first we considered two choices of optical design: a reflector (Cassegrain) or a refractor. The advantage of Cassegrain is its short tube length therefore numbers of satellites adopt this kind of telescopes. However to widen the FoV, the size of secondary mirror becomes much larger which enlarges obscuration ratio higher than $50 \%$. Especially in case of small aperture system, the high obscuration ratio will cause a serious impact to the sensitivity. Moreover the supporting structures of Cassegrain optics is rather complicated making the mechanical design and manufacturing much harder. To maximize the number of source photons within the size of satellite and the cost cap, we chose refraction optics. The allowable maximum diameter of the primary lens becomes $\phi 80$ $\mathrm{mm}$ taking into account the vibration and shock proof lens supporting structures.

\subsubsection{Wavelength band}

The observed wavelength band can be flexibly controlled by the tuning of AR coat, however the achromatic correction of telescope becomes much harder at a wavelength shorter than $230 \mathrm{~nm}$ due to the limited choices of optical materials. We therefore chose $230 \sim 280 \mathrm{~nm}$ band which covers the intermediate band between NUV of GALEX and UVW1 of UVOT/Swift.

In preparation for ray-trace simulation, we defined the observed source spectrum. We therefore assumed a blackbody with a temperature of $T=10^{4} \mathrm{~K}$ emulating SNSBs penetrate through the 5-layer metal-dielectric coating described in Hennessy et al.(2015). ${ }^{14}$ The optimization of the optical design was performed for the wavelength band in which $3-\sigma$ of the penetrated photons are distributed.

\subsubsection{Focal length}

Next we considered the focal length which determines the plate scale. In the aspect of the event rate, the wider FoV is better because the surveyed volume becomes larger. In addition the focal length also constrained by the size of the satellite, the tube length therefore must be shorter than $300 \mathrm{~mm}$. On the other hand the imaging detector has a finite spatial resolution of $12 \mu \mathrm{m}$. To distinguish a transient source from the nearby stars and measure the flux of the object by aperture photometry, the focal length must be longer than a lower limit constrained by the stellar surface density.

Based on the NUV source catalog measured by GALEX, we estimated the stellar surface density in NUV-band in high galactic latitude region. Based on the experiences of ground based telescope ${ }^{15}$ detection limit of point sources is $\sigma \sim 2$, below which even the defacto standard software "Source Extractor" fails detecting sources. Assuming a design goal sensitivity of $20 \mathrm{mag}$ at $\sigma=5$, the magnitude of a star with a confidence level of $\sigma=2$ becomes 21 mag. Then we counted up the stars brighter than 21 mag within the FoV of GALEX and obtained a stellar surface density of $451 \mathrm{deg}^{-2}$. Empirically the aperture photometry method cannot be applied to stellar crowded images with a number density higher than 3000 stars per $10^{6}$ pixels due to the contamination from the nearby objects. Therefore the allowed angular size of a pixel must be smaller than 9.28 arcsec. Consequently the lower limit of the focal length is $267 \mathrm{~mm}$.

\subsubsection{Ray-trace simulation}

Table 3 summarize the specification of the optical design. Within those constraints we optimized the optical system. The resultant telescope consists of 8 elements including two aspherical surfaces to obtaining a wide field of view. Upper panel of Figure 2 shows the ray diagram of the UV telescope. The convex lenses are made of $\mathrm{CaF}_{2}$ while the concave lenses are made of fused silica. In front of the detector a protective glass plate is inserted. The all surfaces of those optical elements are AR coated and the resultant throughput ratio is higher than $90 \%$ in NUV-band.

Lower panel of Figure of 2 shows the spot diagram at various position on the detector. The RMS diameter of the spot is smaller than the pixel size at the field within $20 \mathrm{~mm}$ from the optical axis. At $24 \mathrm{~mm}$ from the axis, the PSF seems somewhat elongated and the RMS spot size is still smaller than the pixel size. At the corner of the detector, 28 34 mm from the axis, the PSF appears distorted asymmetrically and the the PSF spreads over 2 pixels. The detector attached to the optical system physically covers $106 \mathrm{deg}^{2}$ and the Field size of the useful image will be larger than $\sim 60 \mathrm{deg}^{2}$. This means only two-points tiling can cover $100 \mathrm{deg}^{2}$. 
Table 3. Specification of UV Optics.

\begin{tabular}{lr}
\hline \hline Parameter & Value \\
\hline Aperture diameter & $\phi 80 \mathrm{~mm}$ \\
Focal length & $267 \mathrm{~mm}$ \\
F-ratio & 3.34 \\
Lens Construction & 8 elements \\
& (including 2 aspherical lenses) \\
Image circle & $\phi 40 \mathrm{~mm}$ \\
PSF size & $\mu \mathrm{m}(\mathrm{RMS})$ \\
Transmission ratio & $>90 \%$ \\
Plate Scale & 9.27 arcsec \\
Field of View & $\sim 58$ deg $^{2 \dagger}$ \\
Dimensions & $\mathrm{H} 108 \mathrm{~mm} \times$ W 116mm $\times \mathrm{L} 272.5 \mathrm{~mm}$ \\
Weight & $2.6 \mathrm{~kg}$ (Detector and Mount are not included) \\
\hline
\end{tabular}

${ }^{\dagger}$ The detector physically covers $106 \mathrm{deg}^{2}$ and the RMS spot size is smaller than the pixel size in the field of 83.5 $\operatorname{deg}^{2}$.

\subsection{Performance Estimation}

Finally we estimate the detection limit taking into account the optical design shown above. In this calculation we assumed a readout noise of $2 e^{-}(\mathrm{RMS})$ and a dark current of $0.5 e^{-} \mathrm{s}^{-1}$ pixel ${ }^{-1}$ at a temperature of $-20^{\circ} \mathrm{C}$ based on a commercially available BI-CMOS detector. As the external noise sources, we considered the zodiacal light and the earthshine including geocoronal emission lines. Those are the dominant background light in UV-band ${ }^{16}$ and we also considered their optical components may leak through the AR coating, namely "red-leak".

For this calculation we referred the spectral intensities of those sources shown in "STIS Instrument Handbook." ${ }^{17}$ The assumed conditions are the earthshine at $38^{\circ}$ from the limb of the day side of the earth and the relatively high zodiacal light of $m_{\mathrm{V}}=22.1 \mathrm{arcsec}^{-2}$. Thanks to the excellent properties of the AR-coat, the estimated background event rate is lower than 0.07 events s$^{-1}$ pixel $^{-1}$ which is almost negligible comparing with the other noise components. While the dominant noise sources are the dark current and the readout noise of the assumed detector. In this calculation we assumed a single exposure time of $10 \mathrm{~s}$ to avoid blurring due to the unstability of the satellite and cosmic-ray events. Therefore the equivalent noise charge for a combined image for $1800 \mathrm{~s}$ becomes $26.8 e^{-}$(RMS). While the shot noise caused by the dark current is $31.6 e^{-}(\mathrm{RMS})$. Assuming an aperture radius of $1.6 \times$ PSF FWHM (= 1.56 pixel), the total noise charge amounts to $118.9 e^{-}(\mathrm{RMS})$, and the expected detection limit achieves $20.0 \mathrm{mag}(\mathrm{AB})$ at $\lambda=250 \mathrm{~nm}$ assuming a source spectrum of $10^{4} \mathrm{~K}$ blackbody.

It should be noted that the resultant sensitivity is just on the borderline of the mission requirement. There are a lot of possible causes of degradations: increase of dark current, de-focusing due to the temperature variation, disturbance of the satellite attitude, and the other unexpected troubles. To deal with unexpected degradations we should consider the lower operation temperature and further optimization of the specification of AR-coating to increase the signal to noise ratio. In addition the pairing of $\mathrm{CaF}_{2}$ and fused silica cannot compensate the temperature dependent focal-shift in UV-band, amounts to several tens of $\mu \mathrm{m}$ per kelvin. To deal with this inconvenient character, we have to consider adopting a movable focusing stage which can be a failure point.

\subsection{Data Acquisition}

Data acquisition system including the embedded software is the another issue to be considered. The $4 \mathrm{k}$ image detector generates 32 MByte image every $10 \mathrm{~s}$. The data acquisition system must be capable of handling those data stream. Then the data must be reduced and compressed because the narrow bandwidth of satellite does 

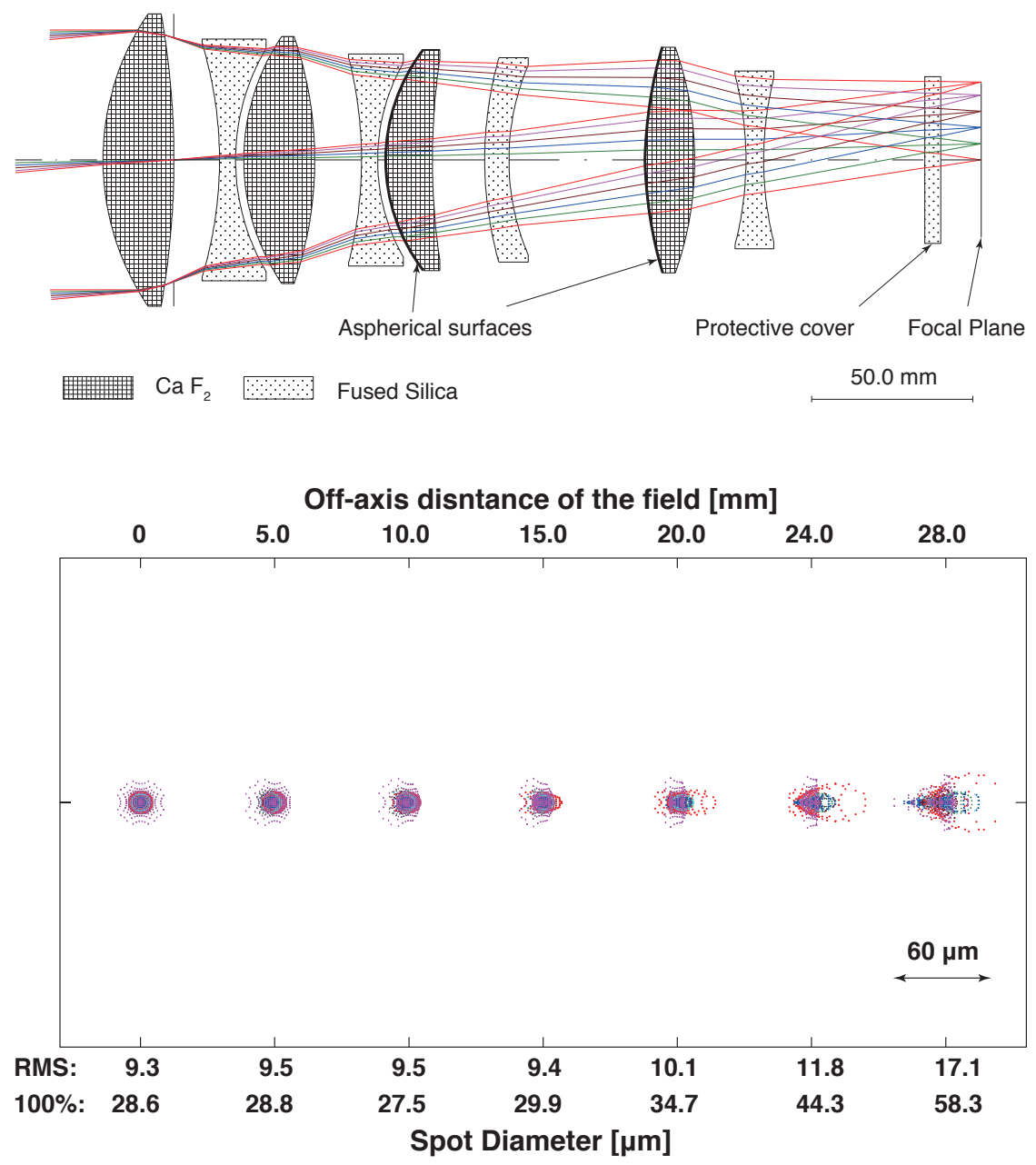

Figure 2. Top - Ray diagram of the UV telescope for $6 \mathrm{U}$ cubesat. That consists of 8 elements (2 groups). The rightmost plate is the protective cover for detector. Bottom - Spot diagrams at various off-axis distances on the focal plane detector surface.

not allow us to downlink whole raw data to the ground. As the required exposure time is longer than $1800 \mathrm{~s}$, the obtained image should be combined to reduce the data size and for rejecting cosmic-rays at the same time. We had already developed a data analysis pipeline for the robotic telescope for GRB follow-up on the ground. The satellite should have simplified version of the pipe-line. Note that the most complicated processes on the ground are treatments of temporally changing weather conditions. In terms of that, the on-board software can be simplified and automatized.

The next step is detection of transient sources by comparing with the UV source catalog or with the observed image just before. Recently, most of observatories on the ground employ the image subtraction method to detecting transient sources, however a high computational cost makes it difficult to use the method in space missions. Therefore we plan to comparing the results of photometry. For those analysis we have developed a high performance on-board computer for a demonstration of real-time image recognition in-orbit launched in 2018. Moreover we also developed software for point source detection, and star identification algorithm for demonstration of star tracker system. Based on those technologies we are designing the data acquisition and analysis system.

If a transient source detected the essential information, flux, coordinate, and pixel values just around the 
transient source is downlinked via the real-time connection. In addition to the transient alert, hourly images will be downlinked via S-band link. Therefore transferred telemetry size becomes $\sim 240$ MByte per day after compression.

\section{SATELLITE SYSTEM}

The satellite is comprised of four subsystem: Communication/Command \& Data Handling (Comm/C\&DH), Attitude Determination and Control system (ADCS), Electric Power system (EPS) and Structure/Thermal. Table 4 summarize the specification of the satellite bus system. To reduce the cost and risk we utilize commercially available qualified cubesat components if available.

\subsection{Comm/C\&DH system}

As the $\mathrm{C} \& \mathrm{DH}$ we must choose a radiation proofed CPU to avoid unexpected troubles caused by single event effects. There are a lot choices of CPU board. For the daily command operation via the Tokyo Tech ground station, the satellite has an UHF/VHF transceiver. In addition, we employ an IRIDIUM transceiver to notify ground facilities when the satellite detects UV transients. This transceiver also support uplink when the GW interferometers detect a GW candidate. The packet size of the short burst data of IRIDIUM is enough to send the coordinate information. Moreover several satellites have already demonstrated the transceiver in space. We can expect connection with the satellite more than once in every 30 minutes. ${ }^{18}$ For telemetry downlink we also employ an S-band transmitter with a bandwidth of $>1$ Mbps.

\subsection{Attitude Determination and Control system}

One of the most challenging system requirements is the attitude stability higher than 10 arcsec during exposure time. In previous satellite project we developed an ADCS by ourselves however the cost efficiency was quite low. To reduce the developing time and suppress the risk of technical trouble, we plan to utilize cataloged ADCS. One of the candidate is produced by Blue Canyon Technology. "XACT" has a 10 arcsec pointing accuracy within a size of $0.5 \mathrm{U}$ and $0.9 \mathrm{~kg}$, satisfies the requirement for both of bus system and science mission.

\subsection{Electric Power System}

The science mission requires $13 \mathrm{~W}$ including a survival heater maintaining the temperature of the optical system and an active cooling system for the imager to reduce the dark current. In addition to the payload the satellite bus system requires $\sim 10 \mathrm{~W}$ in the nominal operation mode and the maximum power consumption of the satellite reached $31 \mathrm{~W}$ during S-band downlink. Assuming a sunshine duration of $60 \mathrm{~min}$ per orbit, the required power generation is $42.1 \mathrm{~W}$, that requires four deployable solar array paddles to secure the power generation. For the stable observation in the night side, the satellite have a $100 \mathrm{Wh}$ Li-ion polymer battery assembly.

\subsection{Structure and Thermal system}

The external dimensions are designed according to the cubesat standard. $\mathrm{CaF}_{2}$ in the telescope is a fragile material therefore the lens support structure was designed very carefully based on the design of Hodoyoshi-1 satellite. As the lens cell structure is sensitive to the external pressure the top-ring of the telescope cannot contact the satellite structure. The main body frame is therefore supported by the dividing panel in order to maintain the spacecraft structure and the telescope, since the main body is hard to secure the rigidity by the enclosure. As for thermal design, part of the main body surface is covered by silver Teflon to appropriately radiate the internal heat. Figure 3 describes the layout of satellite components successfully packed into a $6 \mathrm{U}$ cubesat spacecraft.

\subsection{Ground Stations}

We have developed ground stations for operations of previous small satellite projects. In Tokyo Tech, we have UHF/VHF Yagi antennas for uplink for daily operations and checking housekeeping data. We can also use $\phi 2.4$ $\mathrm{m}$ dish for S-band communication in ISAS/JAXA. We installed a band pass filter and a demodulator for our previous satellite project. We will use the same waveband for which the frequency coordination was already approved. To downink the image data faster we plan to use $\phi 2.4 \mathrm{~m}$ dish in the Singapore station in cooperate with Massachusetts Institute of Technology. Those two S-band stations enable downlink of hourly images. 


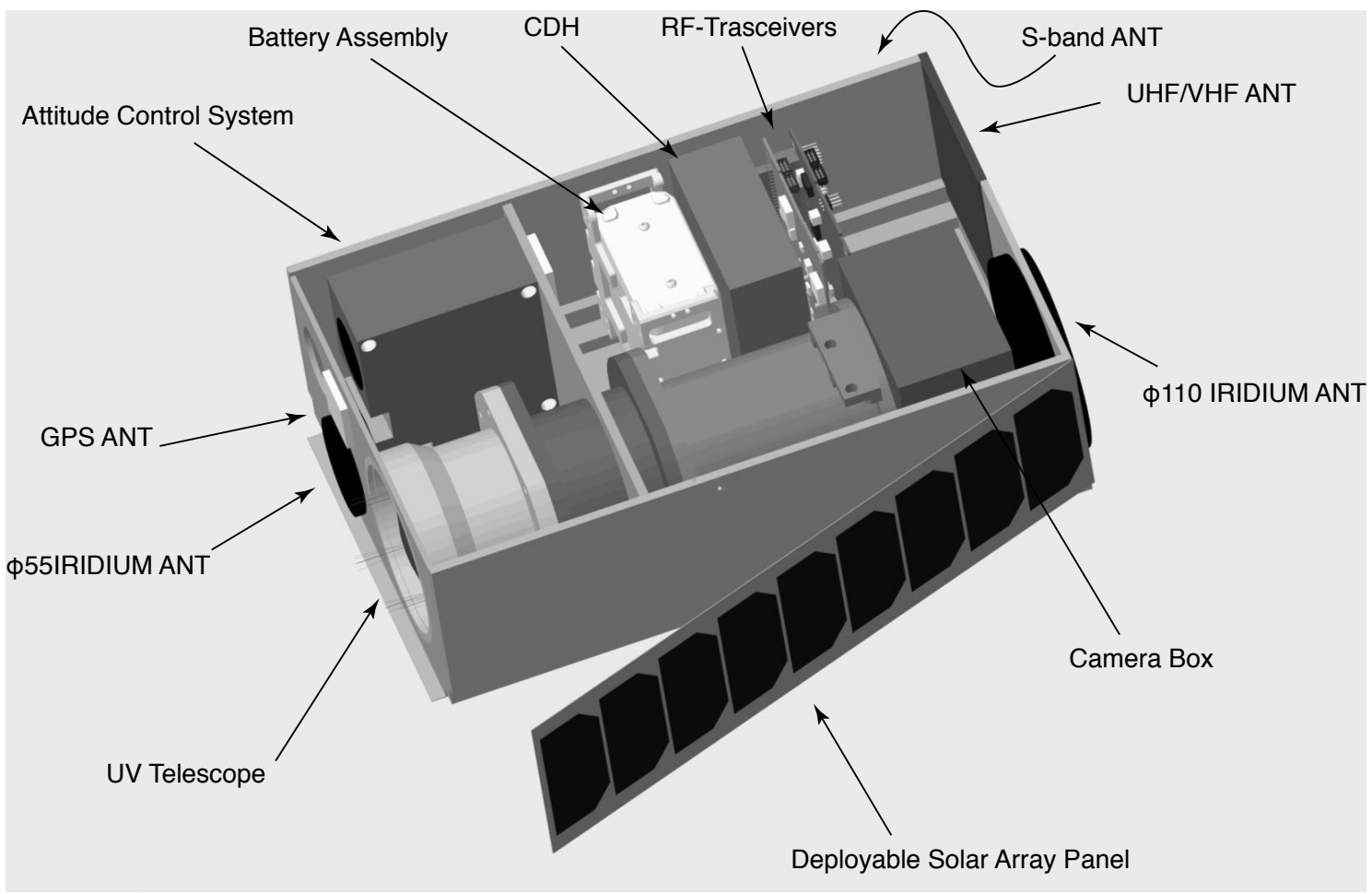

Figure 3. Schematic view of the satellite bus system.

\section{CONCLUSION}

We designed a wide-field UV exploration satellite within constraints of $6 \mathrm{U}$ cubesat to realize the observation mission as soon as possible. Alghough the maximum diameter of the primary lens is rather small, $\phi 80 \mathrm{~mm}$, the advanced detector technologies of the delta-doping and the AR-coat directly deposited on the wafer enable the exploration mission surveying $\sim 60 \mathrm{deg}^{2}$ area at a sensitivity of 20 mag in NUV-band. The expected event rate is $3 \mathrm{SN}$ shock breakouts, 2 tidal disruption events, and as many as Type-Ia SNe, and a lot of stellar flares per year. We can also detect the early phase UV emission from NS-NS mergers discovered by the GW interferometers. The satellite system are designed to be constructed with the commercially available components to reduce the cost and risk. Resultant satellite design can satisfy the mission requirements. The estimated development cost for the entire satellite system is $\sim \$ 500 \mathrm{k}$. We aim to launch the satellite not later than 2022 .

\section{ACKNOWLEDGMENTS}

This work was supported by JSPS Grant-in-Aid for Young Scientists (A) Grant Number JP17H04829. YY thanks Mr. Ryo Adachi of Tokyo Tech for checking the surface stellar density by using data archive of MITSuME telescope.

\section{REFERENCES}

[1] Abbott, B. P. et al., "Gw170817: Observation of gravitational waves from a binary neutron star inspiral," Phys. Rev. Lett. 119, 161101 (Oct 2017).

[2] Tanaka, M., Utsumi, Y., Mazzali, P. A., Tominaga, N., Yoshida, M., Sekiguchi, Y., Morokuma, T., Motohara, K., Ohta, K., Kawabata, K. S., Abe, F., Aoki, K., Asakura, Y., Baar, S., Barway, S., Bond, I. A., Doi, M., Fujiyoshi, T., Furusawa, H., Honda, S., Itoh, Y., Kawabata, M., Kawai, N., Kim, J. H., Lee, C.-H., Miyazaki, S., Morihana, K., Nagashima, H., Nagayama, T., Nakaoka, T., Nakata, F., Ohsawa, R., Ohshima, T., Okita, H., Saito, T., Sumi, T., Tajitsu, A., Takahashi, J., Takayama, M., Tamura, Y., Tanaka, 
Table 4. Summay of satellite bus system.

\begin{tabular}{|c|c|c|}
\hline Parameter & & Value \\
\hline \multirow[t]{2}{*}{ Structure } & Size & $116 \times 239 \times 366 \mathrm{~mm}(6 \mathrm{U}$ cubesat $)$ \\
\hline & Weight & $10 \mathrm{~kg}(\mathrm{TBD})$ \\
\hline \multirow[t]{4}{*}{ Comm } & S-band(down) & QPSK(1 Mbps) \\
\hline & $\mathrm{UHF}(\mathrm{up})$ & $\operatorname{AFSK}(1200 \mathrm{bps})$ \\
\hline & VHF(down) & BPSK(1200 bps) \\
\hline & IRIDIUM SBD & 270 Byte(UP) / 340 Byte(Down) \\
\hline \multirow[t]{4}{*}{ EPS } & Solar cells & InGaP/InGas/Ge (42.1 W @ EOL) \\
\hline & Battery & Li-ion Polymer ( 12.6V / 100Wh) \\
\hline & Main Bus: 12V & Sequential-shunt/non-Stabilized \\
\hline & Power Consumption & $23 \mathrm{~W}$ (Nominal) / $31 \mathrm{~W}$ (Max during S-link) \\
\hline \multirow[t]{2}{*}{ ACS } & Main & non-biased 3 axis control \\
\hline & sub & VSAC (Technical demonstration) \\
\hline \multirow[t]{3}{*}{ Ground Station } & Tokyo Tech & UHF(up)/VHF(down)/IRIDIUM \\
\hline & ISAS/JAXA & S-band $2.4 \mathrm{~m}$ antenna \\
\hline & Singapore/MIT & S-band $2.4 \mathrm{~m}$ antenna \\
\hline \multirow[t]{2}{*}{ Launch } & Launch vehicle & TBD \\
\hline & Orbit & 500 km sun-synchronous (TBD) \\
\hline \multirow[t]{3}{*}{ Management } & Schedule & Not later than 2022. \\
\hline & Total Cost & $\$ 500 \mathrm{k} \sim$ \\
\hline & Mission Life & $>3 \mathrm{yr}$ \\
\hline
\end{tabular}

I., Terai, T., Tristram, P. J., Yasuda, N., and Zenko, T., "Kilonova from post-merger ejecta as an optical and near-Infrared counterpart of GW170817," PASJ 69, 102 (Dec. 2017).

[3] Kasliwal, M. M., Nakar, E., Singer, L. P., Kaplan, D. L., Cook, D. O., Van Sistine, A., Lau, R. M., Fremling, C., Gottlieb, O., Jencson, J. E., Adams, S. M., Feindt, U., Hotokezaka, K., Ghosh, S., Perley, D. A., Yu, P.-C., Piran, T., Allison, J. R., Anupama, G. C., Balasubramanian, A., Bannister, K. W., Bally, J., Barnes, J., Barway, S., Bellm, E., Bhalerao, V., Bhattacharya, D., Blagorodnova, N., Bloom, J. S., Brady, P. R., Cannella, C., Chatterjee, D., Cenko, S. B., Cobb, B. E., Copperwheat, C., Corsi, A., De, K., Dobie, D., Emery, S. W. K., Evans, P. A., Fox, O. D., Frail, D. A., Frohmaier, C., Goobar, A., Hallinan, G., Harrison, F., Helou, G., Hinderer, T., Ho, A. Y. Q., Horesh, A., Ip, W.-H., Itoh, R., Kasen, D., Kim, H., Kuin, N. P. M., Kupfer, T., Lynch, C., Madsen, K., Mazzali, P. A., Miller, A. A., Mooley, K., Murphy, T., Ngeow, C.-C., Nichols, D., Nissanke, S., Nugent, P., Ofek, E. O., Qi, H., Quimby, R. M., Rosswog, S., Rusu, F., Sadler, E. M., Schmidt, P., Sollerman, J., Steele, I., Williamson, A. R., Xu, Y., Yan, L., Yatsu, Y., Zhang, C., and Zhao, W., "Illuminating gravitational waves: A concordant picture of photons from a neutron star merger," Science 358, 1559-1565 (Dec. 2017).

[4] Yatsu, Y., Kawai, N., Matunaga, S., Kimura, S., Matsushita, M., Kawajiri, S., Tawara, K., Ohta, K., Koga, M., et al., "What we learned from the tokyo tech $50 \mathrm{~kg}$-satellite tsubame," in [31st Annual AIAA/USU Conference on Small Satellites], Small Satellite Conference, SSC17-WK-41 (August 2017).

[5] Nikzad, S., Jewell, A. D., Hoenk, M. E., Jones, T. J., Hennessy, J., Goodsall, T., Carver, A. G., Shapiro, C., Cheng, S. R., Hamden, E. T., Kyne, G., Martin, D. C., Schiminovich, D., Scowen, P., France, K., McCandliss, S., and Lupu, R. E., "High-efficiency UV/optical/NIR detectors for large aperture telescopes and UV explorer missions: development of and field observations with delta-doped arrays," Journal of Astronomical Telescopes, Instruments, and Systems 3, 036002 (July 2017). 
[6] Metzger, B. D. and Fernández, R., "Red or blue? A potential kilonova imprint of the delay until black hole formation following a neutron star merger," MNRAS 441, 3444-3453 (July 2014).

[7] Metzger, B. D., Bauswein, A., Goriely, S., and Kasen, D., "Neutron-powered precursors of kilonovae," MNRAS 446, 1115-1120 (Jan. 2015).

[8] Drout, M. R., Piro, A. L., Shappee, B. J., Kilpatrick, C. D., Simon, J. D., Contreras, C., Coulter, D. A., Foley, R. J., Siebert, M. R., Morrell, N., Boutsia, K., Di Mille, F., Holoien, T. W.-S., Kasen, D., Kollmeier, J. A., Madore, B. F., Monson, A. J., Murguia-Berthier, A., Pan, Y.-C., Prochaska, J. X., Ramirez-Ruiz, E., Rest, A., Adams, C., Alatalo, K., Bañados, E., Baughman, J., Beers, T. C., Bernstein, R. A., Bitsakis, T., Campillay, A., Hansen, T. T., Higgs, C. R., Ji, A. P., Maravelias, G., Marshall, J. L., Bidin, C. M., Prieto, J. L., Rasmussen, K. C., Rojas-Bravo, C., Strom, A. L., Ulloa, N., Vargas-González, J., Wan, Z., and Whitten, D. D., "Light curves of the neutron star merger GW170817/SSS17a: Implications for r-process nucleosynthesis," Science 358, 1570-1574 (Dec. 2017).

[9] Ofek, E. O., Rabinak, I., Neill, J. D., Arcavi, I., Cenko, S. B., Waxman, E., Kulkarni, S. R., Gal-Yam, A., Nugent, P., Bildsten, L., Bloom, J. S., Filippenko, A. V., Forster, K., Howell, D. A., Jacobsen, J., Kasliwal, M., Law, N., Martin, C., Poznanski, D., Quimby, R. M., Shen, K. J., Sullivan, M., Dekany, R., Rahmer, G., Hale, D., Smith, R., Zolkower, J., Velur, V., Walters, R., Henning, J., Bui, K., and McKenna, D., "Supernova ptf09uj: A possible shock breakout from a dense circumstellar wind," The Astrophysical Journal 724(2), 1396 (2010).

[10] Tominaga, N., Blinnikov, S., Baklanov, P., Morokuma, T., Nomoto, K., and Suzuki, T., "Properties of Type II Plateau Supernova SNLS-04D2dc: Multicolor Light Curves of Shock Breakout and Plateau," ApJ 705, L10-L14 (Nov. 2009).

[11] Sagiv, I., Gal-Yam, A., Ofek, E. O., Waxman, E., Aharonson, O., Kulkarni, S. R., Nakar, E., Maoz, D., Trakhtenbrot, B., Phinney, E. S., Topaz, J., Beichman, C., Murthy, J., and Worden, S. P., "Science with a Wide-field UV Transient Explorer," AJ 147, 79 (Apr. 2014).

[12] Pigulski, A., Baran, A., Bzowski, M., Cugier, H., Czerny, B., Daszyńska-Daszkiewicz, J., Dziembowski, W., Handler, G., Kołaczkowski, Z., Królikowska, M., Krzesiński, J., Maciejewski, G., Michalska, G., MolendaŻakowicz, J., Moskalik, P., Niedzielski, A., Niemczura, E., Ostrowski, J., Pamyatnykh, A., Ratajczak, M., Rucinski, S., Siwak, M., Smolec, R., Szutowicz, S., Tomov, T., Wyrzykowski, Ł., Zoła, S., and Sarna, M., "UVSat: a concept of an ultraviolet/optical photometric satellite," in [Second BRITE-Constellation Science Conference: Small satellites-big science], Proceedings of the Polish Astronomical Society 5, 76-81 (Sept. 2017).

[13] Murthy, J., "The diffuse ultraviolet foreground," Ap\&SSS 349, 165-169 (Jan. 2014).

[14] Hennessy, J., Jewell, A. D., Hoenk, M. E., and Nikzad, S., "Metal-dielectric filters for solarblind silicon ultraviolet detectors," Appl. Opt. 54, 3507-3512 (Apr 2015).

[15] Yatsu, Y., Kawai, N., Shimokawabe, T., Vasquez, N., Ishimura, T., Kotani, T., Yanagisawa, K., Yoshida, M., Nagayama, S., Shimizu, H., Toda, H., and Kuroda, D., "Development of MITSuME-Multicolor imaging telescopes for survey and monstrous explosions," Physica E Low-Dimensional Systems and Nanostructures 40, 434-437 (Dec. 2007).

[16] Murthy, J., "Observations of the near and far ultraviolet background," Ap\&SS 320, 21-26 (Apr. 2009).

[17] Riley, A. et al., [STIS Instrument Handbook, Version 17.0] (May 2018).

[18] YAMADA, K., MORIYOSHI, T., MATSUMARU, K., KANEMARU, H., ARAYA, T., SUZUKI, K., IMAMURA, O., AKITA, D., NAGATA, Y., SHOJI, Y., TAKAHASHI, Y., WATANABE, Y., ABE, T., et al., "Re-entry Nano-Satellite with Gossamer Aeroshell and GPS/Iridium deployed from ISS," in [Proceeding of The 25th Workshop on JAXA Astrodynamics and Flight Mechanics, held July 27-28, 2015, ISAS/JAXA, Sagamihara, Japan, 2017-f-021], 25, 2017-f-21 (July 2015). 